\title{
7
}

\section{Structure-Function Relationship of DNA Repair Proteins: Lessons from BRCA1 and RAD51 Studies}

\author{
Effrossyni Boutou ${ }^{1,2}$, Vassiliki Pappa ${ }^{3}$, \\ Horst-Werner Stuerzbecher ${ }^{4}$ and Constantinos E. Vorgias ${ }^{1}$ \\ ${ }^{1}$ Department of Biochemistry \& Molecular Biology \\ Faculty of Biology, School of Sciences Athens University, Athens \\ ${ }^{2}$ Prenatal Diagnosis Lab, Laiko Hospital, Athens \\ ${ }^{32}{ }^{\text {nd }}$ Propaedeutic Pathology Clinic, Medical School, Athens University, Athens \\ ${ }^{4}$ Molecular Cancer Biology Group, Institute of Pathology, UK-SH, Luebeck \\ 1,2,3Greece \\ ${ }^{4}$ Germany
}

\section{Introduction}

Accurate transfer of genetic information is vital for all living organisms in order to guarantee species survival. DNA damage occurs spontaneously during a cell's life due to either endogenous causes such as Reactive Oxygen Species (ROS) produced during metabolism or due to exogenous insults such as Ionizing Radiation (IR) or genotoxic agents in food / water and environment, to which an organism is exposed. Endogenous damage, due to intrinsic instability of chemical bonds in DNA structure, occurs spontaneously under normal physiologic conditions and is calculated to be approximately $10^{4}$ events per cell, per day (Lindahl, 1993). Moreover, during DNA replication base adducts can cause collapse of replication forks and DNA double strand breaks (DSBs) are introduced in order to reinitiate genome duplication process.

As the genome carries all necessary information for life and evidently preservation of genome integrity is critical for cell survival, a number of mechanisms have evolved over time to ensure the most effective performance of the genome repair procedure. DNA repair mechanisms are capable of repairing practically all different types of chromosomal lesions (single and double strand breaks, base modifications, etc.) ensuring that genetic information is accurately transferred to the next generation. The cell's response to DNA damage (DNA Damage Response, DDR) encompasses a complex network of proteins, consisting of DNA damage recognition, signal transduction, transcriptional regulation, cell cycle control, DNA repair and verification of the repair efficiency, depending on the type of lesion, the replication status of the genome as well as the cell cycle stage. (scheme 1). Many excellent recent reviews as well as other chapters in the current volume extensively cover this topic (Rogakou, 1999; Lisby \& Rothstein 2005; Murphy \& Moynahan, 2010).

Defects in repair efficiency are the consequence of dysfunction of either upstream damage signalling or the central repair process. The current chapter covers topics referring to 


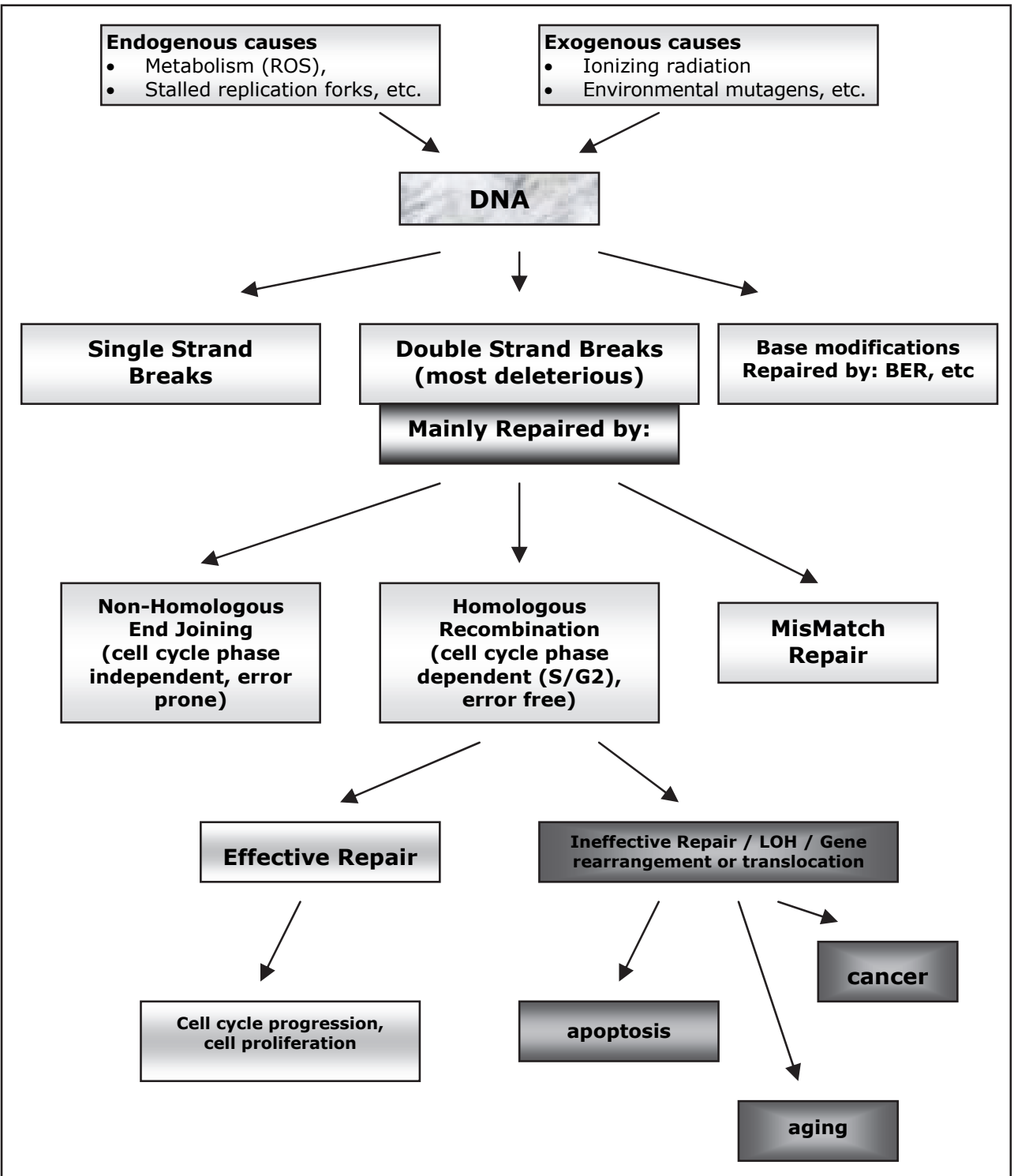

Scheme 1. Simplified diagram of DNA damage response network

factors/events influencing the structure - function relation of key molecules involved in each of the two processes, namely the BRCA1 and RAD51/Rad51 proteins, respectively. The breast cancer susceptibility gene 1 (brca1), isolated by reverse genetics in 1994, encodes for a large multifunctional protein (BRCA1) whose function is regulated by multiple posttranslational modification events, driving the multi tasks performed, by which BRCA1 conducts almost all steps of DDR. The important anti-tumorigenic role of BRCA1 is strongly 
supported by its correlation with increased breast \& ovarian familial cancer susceptibility in individuals carrying BRCA1 mutations. On the other hand, RAD51 is a relatively small and rigid protein playing a basic role (homology search strand and strand exchange) in the high fidelity DNA repair mechanism of Homologous Recombination (HR). RAD51 appears absolutely vital for cell survival, as its depletion results in embryonic lethality, it is highly conserved throughout evolution and up to now there is not a single mutation in the amino acid sequence detected in any type of cancer, although there is a strong correlation between its expression levels and both cancer development and cancer progression.

\section{Consequences of genomic instability}

Loss or insufficiency of DDR and genome repair can lead to an increased susceptibility to cancer due to the consequential genomic instability. Ineffective repair may result in subsequent mutations of genes required for cellular replication and division. The genome repair pathways also communicate with processes involved in induction of senescence and apoptosis when the damage cannot be repaired. Carefully balanced signalling cascades and regulatory systems are implicated in the maintenance of healthy cell survival in order to unfavour tumorigenesis and maintain stem and progenitor cells for renewal (anti-ageing) (Seviour \& Lin 2010). Therefore, an effectively repaired genome is crucial not only for cancer prevention but also for lifespan extension. This notion is even more enhanced by the emerging benefit of the response of HR defective tumors to double strand break (DSB) producing therapies a promising and continuously evolving field. A clearer understanding of the biochemical, structural and genetic processes in conjuction with clinical data will lead to the development of more effective treatment strategies for both cancer and ageing processes.

\subsection{Genomic instability and cancer}

It is generally accepted that tumors are derived from a single genetically unstable cell, and that the unstable cell population as a whole continues to acquire further chromosomal abnormalities over time, although the precise mechanisms of acquisition of these abnormalities still remain unclear. Hereditary cancers are often characterized by the presence of a specific type of genomic instability, termed chromosomal instability. In these cancers, chromosomal instability can often be attributed to mutations in DNA repair genes, suggesting that the driving force behind tumor development is an increase in spontaneous genetic mutations resulting from lack of appropriate management of DNA damage. A second form of genomic instability, termed microsatellite instability, is also associated with defects in DNA repair, namely the mismatch repair system. However, in non-hereditary sporadic tumors, the picture is less clear. It should be emphasized that cancer is an extremely complex set of diseases, and that cancer cells develop many different mechanisms to achieve a similar phenotype of independent and uncontrolled growth (Hanahan \& Weinberg, 2000; Luo et al., 2009 as reviewed by Schild \& Wiese 2010).

Many of the DDR components including BRCA1 are known to be lost or mutated in human tumors. While loss of BRCA1 has been shown to lead to the development of mammary tumors in mouse models, the genetic diversity within those tumors suggests that the loss of BRCA1 may not directly be responsible for tumorigenesis. It is more likely, therefore, that the role of BRCA1 in the initiation of cancer is a result of its effects on DNA repair and the maintenance of genomic integrity. BRCA1 -/- tumors are shown to display numerous 
chromosomal aberrations. Analysis of BRCA1 -/- mouse models, coupled with the study of human BRCA1 -/- tumors, has revealed prevalence for p53 mutations in these tumors, which is likely to be caused by the decrease in genomic stability associated with the defects in DNA repair. Overall, these and many other data suggest that the loss of cell cycle checkpoints confers a selection advantage to cells with DNA repair defects, thereby triggering tumorigenesis in genetically unstable cells. Moreover, an increase in genomic instability is significantly correlated with the metastatic potential of the tumor. Further studies are required to determine whether this involvement in metastasis is a result of acquired genetic mutations resulting from DNA repair defects, or whether other mechanisms are required for this process (Murphy \& Moynahan, 2010).

DNA repair by the high fidelity mechanism of homologous recombination, termed as Homologous Recombinational Repair (HRR) is practically the only 'error free' repair mechanism of the cell and as it requires a sister chromatid, normally is active in late S and G2 phases of the cell cycle. HRR involves a compex network of recombination mediators and comediators. Defects in recombination mediators and co-mediators, leading to impaired HRR, are indicated as major contributors in carcinogenesis and particularly in breast cancer (reviewed by Pierce et al., 2001; Henning \& Stuerzbecher, 2003; Murphy \& Moynahan, 2010). Nevertheless, up to now not a single mutation in the coding region of RAD51, the central recombinase in the HRR pathway, has been found in many tumor types examined. However, many primary tumor cells and cancer cell lines express significantly modified levels of RAD51 (Maacke et al., 2000; Henning \& Stuerzbecher 2003; Klein 2008) and at least partly, this misregulation in protein expression levels is correlated with the polymorphism G->C in the 5 'untranslated region of rad51 mRNA, as shown in some cases of hereditary breast tumors with BRCA2 mutation. As extensively discussed in the excellent and comprehensive review of Schild \& Wiese 2010, RAD51 overexpression presumably complements initial HRR defects, thereby limiting genomic instability during carcinogenic progression and may explain the high frequency of TP53 mutations in human cancers, as wild-type p53 represses RAD51 expression. Notably, both positive and negative regulations of HRR are required to maintain genomic stability by precise repair and suppression of deleterious rearrangements.

\subsection{Genomic instability and ageing}

DNA damage is a prominent cause of cancer in frequently dividing cells since cell proliferation is a prerequisite for the manifestation of genetic changes as permanent mutations. In contrast, DNA damage in infrequently dividing cells is likely a prominent cause of ageing (Best, 2009). Therefore, in addition to its role in the maintenance of genomic integrity, the DDR has been hypothesized to play a critical role in organismal ageing. Supporting to this hypothesis is the observation that DNA repair disorders such as Werner's syndrome, Bloom's syndrome and Ataxia telangiectasia, syndromes also characterised by premature ageing and / or retarded growth, are often called "segmental progerias" ("accelerated ageing diseases"). Individuals suffering from such diseases appear elderly and suffer from ageing-related diseases at an abnormally young age, while not manifesting all the symptoms of old age.

Ageing, resulting from the accumulation of damage to molecules, cells, organs and tissues over time, is believed to be caused by two cellular processes: senescence and apoptosis.

\subsubsection{Senescence and DNA damage}

Senescence, a phenomenon describing the irreversible cease of cell division, was initially described by Hayflick and Moorhead in 1961 and includes replicative senescence and oncogene-induced senescence, both of which involve aspects of the DDR. 
Replicative senescence results from progressive shortening of telomeres with repeated rounds of cell replication. p53-dependent senescence serves as a tumor suppressor mechanism and is activated by the uncapping of critically shortened telomeres which are recognised as damaged DNA (Feldser and Greider, 2007). Recent studies argue that p53 can either activate or suppress senescence in cells, depending on their specific transcriptional activities and its interaction with partner molecules. As described by Vigneron \& Vousden, 2010, the role of p53 in cell fate determination is even more complex as it involves epigenetic modifications of chromosomal DNA and relates chronic DDR signalling with increased levels of p53 acetylation. In addition to p53 other DNA damage response proteins like ATM have been associated with replicative senescence. ATM depletion in mice results in an increase in both chromosomal end-to-end fusion events and cell cycle-dependent telomere loss. These mice exhibit a premature ageing phenotype as defined by increased hair graying, alopecia and marked weight loss. Expression of mutant BRCA1 in mice also results in premature ageing, accompanied by an increase in cellular senescence and an increased susceptibility to certain cancers. The enhanced senescence observed in these mice may interfere with the fact that senescent cells have been noted to modify their tissue microenvironment. This phenomenon is thought to synergize with the accumulation of DNA damage over time to encourage cancer growth.

Oncogene-induced senescence can be induced by the overexpression of oncogenes by among others the induction of DNA damage resulting from both the generation of reactive oxygen species (ROS) and the hyper-replication of DNA. Both of these mechanisms activate the DNA damage response, which result in senescence by similar processes that induce replicative senescence.

\subsubsection{Apoptosis and DNA damage}

The accumulation of DNA damage can also lead to apoptosis. Activation of p53 by DNA damage and its role in the regulation of expression of pro-apoptotic proteins has been well documented. This is further supported by the fact that functional p53 is not detected to the majority of tumors. In cases of decline of the immune system an increase in p53 mediated apoptosis has been observed, linking ageing with apoptotic function. Moreover, constitutively activated p53 in mice also showed that, while high levels of p53 protect against cancer, it also accelerates the ageing process by reducing the mass of various tissues. The human condition Ataxia telangiectasia, which results from mutations in ATM, is associated with substantial neuro-degeneration. This has been shown in a mouse model to result from an accumulation of neurons harboring genomic damage, due to the inability of the mutant ATM protein to stimulate the p53 apoptotic cascade. Chk2 has also been shown to regulate apoptosis in a p53-dependent manner in vitro and in vivo in response to DNA damage (Seviour \& Lin, 2010). Notably, the major recombinase of HR, Rad51, seems also to interact with p53, possibly serving as a tool for monitoring the extension as well the effectiveness of DNA repair processes (Henning \& Sturzbecher Toxicology, 2003; Morita et al., 2010).

Consequently, impaired DDR appears to have dramatic effects on both tumorigenesis and premature ageing. At the molecular level, DDR impairment could be attributed to irregular interactions between the complexes involved in each process due to structural changes of DDR components resulting from either mutagenesis, modified post-translational modifications of altered protein levels, guiding equilibrium in favour of abnormal decisions. 


\section{Structural \& functional integrity is essential for protein interactions with partner molecules}

The efficient performance of DNA repair processes requires the coordinated actions of many players and mainly depends on proper interactions between the protein components of each pathway involved which in turn mainly relies on their functional structure (structure function relationship). At least three major mechanisms influence protein function due to or independently of its tertiary structure:

a. Missense mutations in the coding region can modify the primary structure of a protein resulting in dys-functional folding of the protein, and / or instability in the cellular environment.

b. Post-translational modifications (phosphorylation, ubiquitination, ribosylation and acetylation) regulating distinct interactions with partner molecules driving the various pathways in which the protein in question is implicated.

c. Regulation of the protein levels, availability through regulation of the quantity of the protein, which can drive cell decisions in improper pathways, leading to abnormal cell cycle progression, cell division and possibly malignant transformation or aggressive tumor progression.

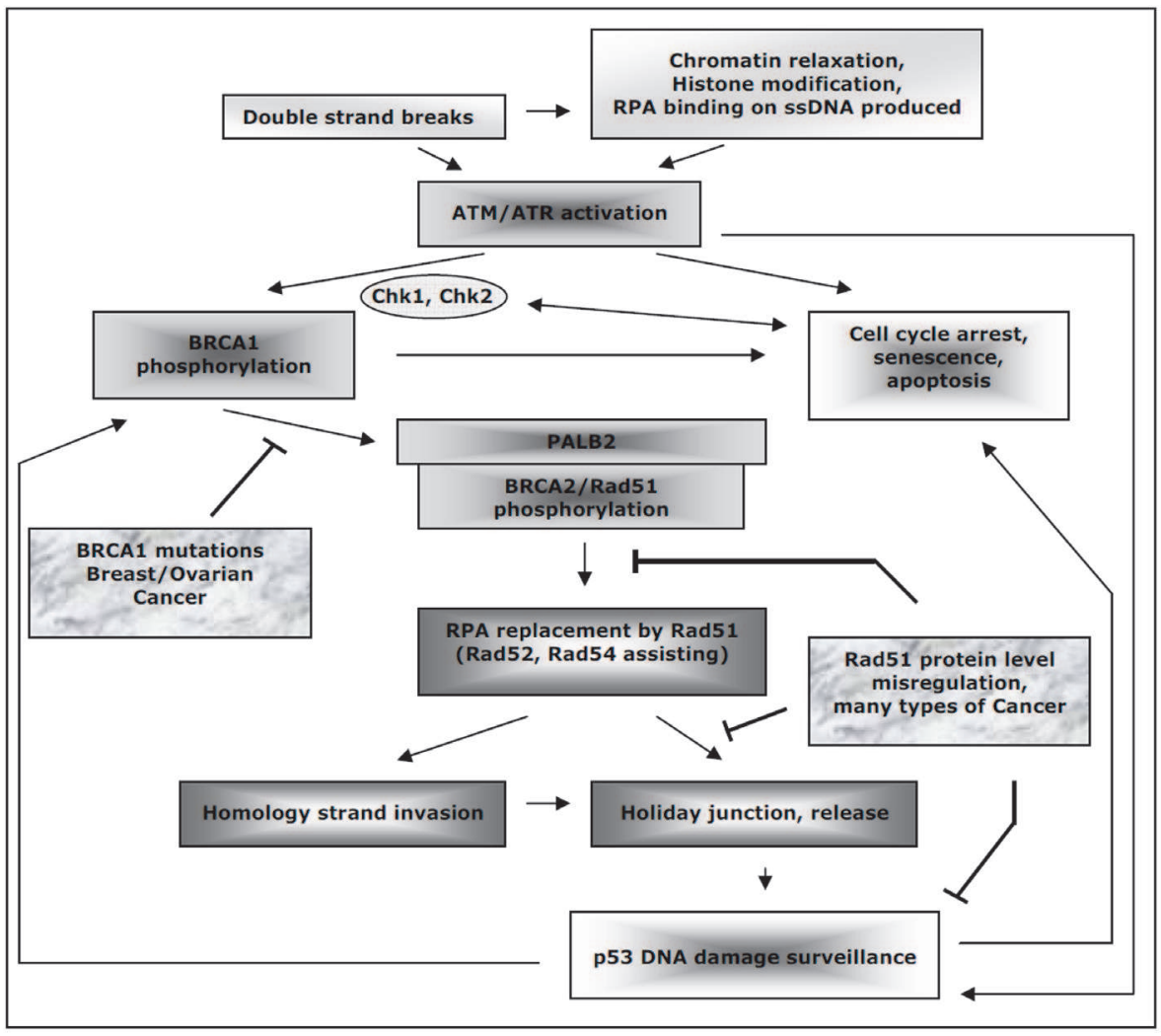

Scheme 2. Simplified Scheme of major steps of DNA Double strand break repair by Homologous Recombination. 
The fundamental proteins involved in the HRR pathways are highly conserved in almost all organisms ranging from bacteria to human. The significance of this repair system is also indicated by the fact that defects in HRR cause human hereditary cancers as well as sporadic tumors. In many cases the dysfunction of proteins observed in many tumors helps to elucidate all three categories of the mechanisms mentioned above and to clarify different aspects of DDR pathways (Murphy \& Moynahan, 2010).

Herein we will focus on current information regarding the structure - function relationship of two key players in regulation and performance of DSB repair - the most deleterious reported lesion of the genome - BRCA1 and RAD51. BRCA1 is a core component of many multi-molecular complexes involved in DNA damage detection, HR regulation, cell cycle regulation and genome transcription. RAD51, a key factor of HRR, replaces RPA on the produced single strands of damaged DNA and performs the search for homologous DNA strand and exchange in order to restore the damaged DNA sequence according to its sister chromatid. Moreover, RAD51 is implicated in telomere maintenance via ALT pathway and is also involved in mitochondrial DNA repair. Via at least its indirect interaction with BRCA1 as well as with direct p53 complex formation, RAD51 seems to be an interplayer responsible for communication between DNA repair effectiveness, cell replication, apoptosis or senescence decisions.

\subsection{The BRCA1 structure - function relation paradigm}

The Breast Cancer Susceptibility Gene 1 protein (BRCA1) is a multifunctional nuclear phosphoprotein of 1863 residues $(220-240 \mathrm{kDa})$. BRCA1 was attributed the role of a tumor suppressor involved in multiple cellular functions (Starita \& Parvin, 2003). Most of BRCA1 is located in the cell nucleus and is phosphorylated in a cell cycle-dependent manner by a number of kinases (reviewed by Ouchi, 2006). Depending on the position and the number of phosphorylated residues, BRCA1 participates in different multiprotein complexes performing diverse tasks. Therefore, BRCA1 has been implicated in a variety of functions required for the maintenance of genomic stability (Rowling et al., 2010).

Regarding DDR, BRCA1 has been attributed many roles in regulation of genome integrity including DNA replication, cell cycle checkpoint control, apoptosis, regulation of transcription, chromatin unfolding and protein ubiquitination. The ascribed functions are exerted through an extensive number of protein interactions reported (Jasin M. 2002 as cited in Murphy \& Moynahan 2010). In brief, upon detection of chromatid relaxation due to breakage of both strands of the double helix of DNA, BRCA1 - being activated by ATR kinase - is recruited to the damage breakpoint assisting assembly of the BRCA2 - RAD51 complex in order to replace RPA and restore damage by the high fidelity process of HRR. In parallel, BRCA1 interaction with Fanconi Anemia (FA) and other complexes regulates G1/S and G2/M checkpoints. BRCA1 implication in cell cycle regulation is assisted by complex formation with BRCA1 interacting protein C-terminal helicase (BRIP1) and CtIP which are activated in S-phase by post-translational modifications. A graphical representation of the BRCA1 protein, including sites of both post-translational modifications and regions involved in protein-protein interactions, is depicted in Fig 1.

The amino-terminal region of BRCA1 contains a distinct $~ 100$ aa RING finger motif involved in ubiquitin ligase activity and enables BRCA1 to mono- or poly-ubiquitinate cellular proteins. BARD1 (another RING and BRCT domain-containing protein) is the 'permanent' partner of BRCA1 in the formation of the ubiquitine ligase complex. Phosphorylation of specific residues of BRCA1 appears to regulate its participation in transcription regulation and ubiquitination of substrate proteins. As many different BRCA1 species are produced by alternative splicing of its mRNA, the phosphorylated residues each form contains may 
regulate different functions. Moreover, the balance between full length and spliced forms of BRCA1 may play an important role in tumor suppression (Ouchi, 2006).

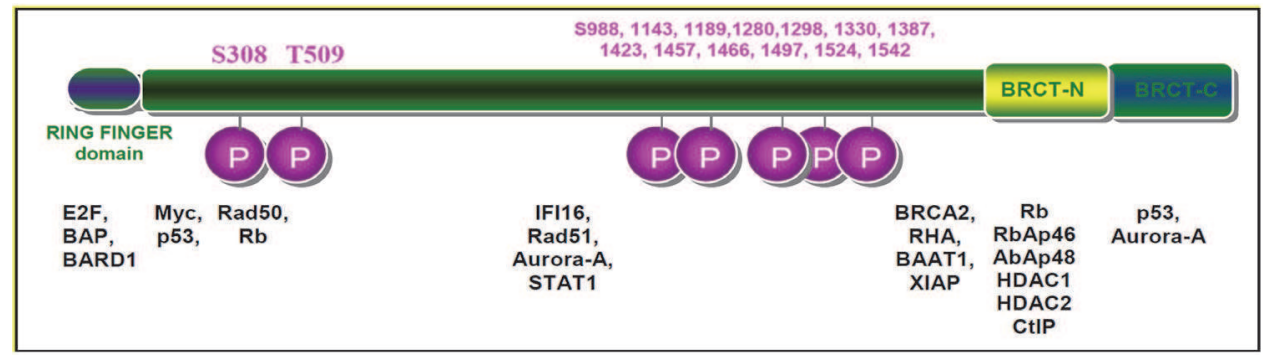

Fig. 1. Primary structure of BRCA1 Tumor Suppressor Protein. The phosphorylation sites and and its binding partners are indicated.

The carboxy-terminal domain of BRCA1 contains two structurally identical BRCT (BRCA1 C-terminal) tandem repeats each containing $\sim 90$ amino acid residues. BRCT domains are found in proteins involved in DNA repair and maintenance of genomic stability, and more recently, the BRCT repeat has been recognized as a phosphopeptide-binding domain. The structure of each repeat consists of a parallel four-stranded $\beta$-sheet located at the central part of the domain surrounded by three a-helices (Fig. 2). The two BRCT repeats fold together in a specific head-to-tail manner, giving rise to the formation of a conserved, almost allhydrophobic, inter-repeat interface, forming a phosphopeptide binding pocket. BRCT like domains have also been found in BRCA1 interacting proteins such as 53BP1 and BARD1. BRCT repeats are a family of phosphopeptide binding domains implicated in DNA damage response. Therefore, BRCTs are considered as protein-docking modules involved in eukaryotic DNA repair. Although BRCTs are characterized by low sequence homology they retain a generally well-conserved structure organization.

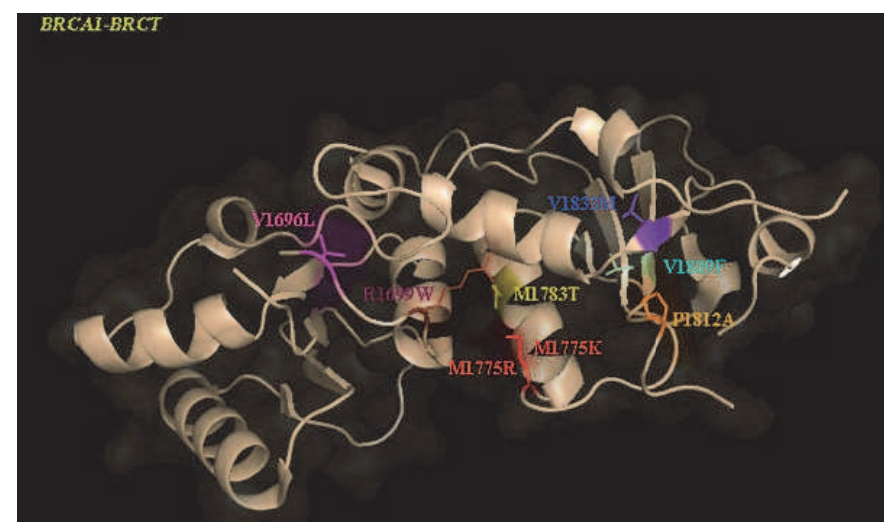

Fig. 2. Ribbon representation of the BRCA1-BRCTstructure. Positions of selected cancerrelated mutations are indicated. M1775K and M1783T are located at the inter-BRCT-repeat interface where the BRCA1-BRCT binding groove for Phe 13 is also located. The exposed V1696L is located at the N-terminal BRCTstructural repeat. V1809F, P1812A are found at the C-terminal BRCT repeat. The positions of missense mutations from previously published studies are also depicted. (From Drikos et al., 2009). 
Analysis of the BRCA1 mutational database (BIC, http://research.nhgri.nih.gov/bic/) indicates that both RING and BRCT repeats are most frequently mutated in women at risk of cancer, and have been further studied. Many research groups have used structural and biochemical methods to probe the function of BRCA1 and characterize the plethora of unclassified variants identified in breast cancer patients found in BIC database. Among the hundreds of distinct mutations uncovered in BRCA1, for the vast majority, there is insufficient genetic linkage data to determine the cancer risk associated with them (Glover, 2006).

The central region of BRCA1, between the two terminal domains, bears relatively low sequence identity between mammalian BRCA1 homologs, and attempts to define structured domains within this region indicate that this part of BRCA1 is largely unstructured (Glover, 2006; Mark, 2005). This region is extensively phosphorylated by DNA damage-associated kinases like ATM and may serve as a phosphorylation-dependent docking site for other proteins involved in the DNA damage response, or even for damaged DNA itself (Paul et al., 2001; Mark, 2005; Ouchi, 2006).

\subsection{BRCA1 structure modifications found in cancer}

Mutations in brca1 and brca2 genes have been found in $30-50 \%$ of hereditary breast and ovarian cancers. Women carrying BRCA1 mutations are particularly susceptible to the development of breast or ovarian cancer at an age earlier than 35-40 years old with a probability rate of $45-60 \%$ and $20-40 \%$, respectively.

Most cancer-associated BRCA1 mutations identified so far, result in the premature translational termination of the protein and influence BRCA1 integrity and function. A large number of missense mutations is located in BRCT tandem repeats of BRCA1, while only few of them may cause loss of the protein's function, abolition of protein interactions and protein miss-localization. Therefore, it seems that the BRCT repeats in BRCA1 are essential for the tumor suppressing function of the protein as protein truncation and missense variants within the BRCT domain have been shown to be associated with human breast and ovarian cancers.

Variants that result in large truncations are deleterious to function and therefore can be classified as disease-associated. In contrast, missense mutations typically remain unclassified. Thus, the BIC database currently contains more than 108 missense mutations in the BRCT domains of BRCA1, but only $7 \%$ of them have been classified. These missense mutations may be either polymorphisms or mutations predisposing the carrier to cancer progression. The variants D1692Y, C1697R, R1699W, A1708E, S1715R, P1749R and M1775R all appear to be associated with an increased risk of breast cancer, while M1625I appears to be a benign polymorphism (Williams et al., 2003). Unfortunately, most of the missense mutations could not been assessed for disease association. An attempt to classify these variants by measuring the thermodynamic stability of the BRCA1 BRCT domains resulted in investigation of the effects of 36 missense mutations (Rowling et al., 2010). The mutations show a range of effects. Some do not change the stability, whereas others destabilize the protein by as much as $6 \mathrm{kcal}$ mol-1; one-third of the mutants were considered to destabilize the protein by an even greater amount, as they could not be expressed in soluble form in Escherichia coli. Several computer algorithms were used in an attempt to predict the mutant effects. According to these results the variants were grouped into two classes (destabilizing by less than or more than $2.2 \mathrm{kcal}$ mol-1). Importantly, with the exception of the few mutants located in the binding site, none showed a significant reduction in affinity for phosphorylated substrate. These results indicate 
that despite very large losses in stability, the integrity of the structure is not compromised by the mutations. Thus, the majority of mutations seem to cause loss of function by reducing the proportion of BRCA1 molecules that are in the folded state and increasing the proportion of molecules that are unfolded. The authors predict that small molecule stabilization of the structure could be a generally applicable preventative therapeutic strategy for rescuing many BRCA1 mutations. Another recent approach by Lee et al., 2010, extended in 117 variants, comprehensively shows how functional and structural information can be useful in the development of models to assess cancer risk.

Cancer-associated mutations in the BRCT domain of BRCA1 (BRCA1-BRCT) abolish its tumor suppressor function by disrupting interactions with other proteins such as BACH1. Many cancer-related mutations do not cause sufficient destabilization to lead to global unfolding under physiological conditions, and thus abrogation of function probably is due to localized structural changes. Molecular dynamics simulations on three cancer-associated mutants, A1708E, M1775R, and Y1853ter, and on the wild type and benign M1652I mutant, followed by comparison of the structures and fluctuations showed that only the cancerassociated mutants exhibited significant backbone structure differences from the wild-type crystal structure in BACH1-binding regions, some of which are far from the mutation sites. These BACH1-binding regions of the cancer-associated mutants also exhibited increases in their fluctuation magnitudes compared with the same regions in the wild type and M1562I mutant, as quantified by quasiharmonic analysis. The increased fluctuations in the diseaserelated mutants suggest an increase in vibrational entropy in the unliganded state that could result in a larger entropy loss in the disease-related mutants upon binding BACH1 than in the wild type. Vibrational entropies of the A1708E and wild type in the free state and bound to a BACH1-derived phosphopeptide, calculated using quasiharmonic analysis, determined the binding entropy difference DeltaDeltaS between the A1708E mutant and the wild type. In overall such biophysical/biochemical studies supported by suited algorithms showed that the observed differences in structure, flexibility, and entropy of binding are likely to be responsible for abolition of $\mathrm{BACH} 1$ binding, and illustrate that many disease-related mutations could have very long-range effects. Such methods have potential for identifying correlated motions responsible for other long-range effects of deleterious mutations. (Gough et al., 2007)

The C-terminal BRCT domains are also evidenced to mediate the transcriptional activity of BRCA1. Most of the published mutations within the BRCT domains have been reported to affect BRCA1 nuclear functions including DNA repair and transcriptional activity. The biochemical and biophysical studies of our group have already demonstrated that mutations of the BRCT domain: (i) affected the folding of the domain to a varying degree depending on the induced destabilization and (ii) altered and abolished the affinity of BRCT domain to synthetic phosphopeptides corresponding to BRCT interacting regions of pBACH1/ BRIP1 and pCtIP, by affecting the structural integrity of the BRCT active sites.

BRCA1 is a nuclear-cytoplasmic shuttling protein and its nuclear localization is regulated by the combined action of nuclear localization (NLS) and nuclear export signals (NES). In most cases, however, cellular and ectopically expressed BRCA1 are primarily nuclear due to nuclear import mediated by the two NLSs and interaction with the RING domain binding protein, BARD1, which can carry BRCA1 into the nucleus and trap it there by masking its nuclear export signal.

Despite the structural studies of BRCA1-BRCT protein mutants, the influence of these mutations at protein localization in cellular level has not yet been adequately addressed. Only few of them have already determined to present protein mislocalization. BRCA1 mutations of 
the BRCT domain altered BRCA1 localization, causing the protein to be excluded from the nucleus. Two of the C-terminal mutations (M1775R and Y1853X) that restricted nuclear localization are identical to mutations that disrupt BRCA1 C-terminal folding, suggesting that the conformational changes they elicit might be deleterious to BRCA1 nuclear transport. This nuclear exclusion was not due to increased nuclear export, but to reduced nuclear import. Similar findings were observed for both the overexpressed and endogenous forms of the BRCT mutant, BRCA1 (5382insC). Also, Chen et al., 1995 have published controversial findings, which claimed that BRCA1 was detected almost exclusively in the cytoplasm in breast cancer tissues, but remained nuclear in normal tissue and in other cancer cell types.

In our laboratory more than fifteen BRCA1-BRCT proteins mutants have already been studied for structural and functional alterations in protein's integrity. The most destabilizing protein mutants such as M1775K, V1809F (Fig3) were collected in order to be examined in cellular level about their impact in BRCA1 subcellular compartmentalization. M1775K is a rare breast cancer-linked mutation and it has been identified only in two unrelated families of European ancestry with a history of breast cancer. Met1775 is strongly involved in the phosphopeptidebinding pocket of the BRCT domain. The mutation of Met1775, namely the mutation M1775R, is much more frequent worldwide among patients with hereditary breast/ovarian cancer, its association with the disease is epidemiologically established and was the first characterized to be linked to cancer. The M1775R mutation has already shown to change the intracellular localization of BRCA1 protein which is less focused into the nucleus. The M1775K missense variant according to our in vitro experiments fails to bind to synthetic peptides such pBACH1/BRIP1 or pCtIP. Structural analysis of the interatomic interactions of Lys1775 show a direct clash of its side chain with Phe 13 of either phosphopeptide, a result arising from the disruption of the BRCT-phosphopeptide binding pocket.

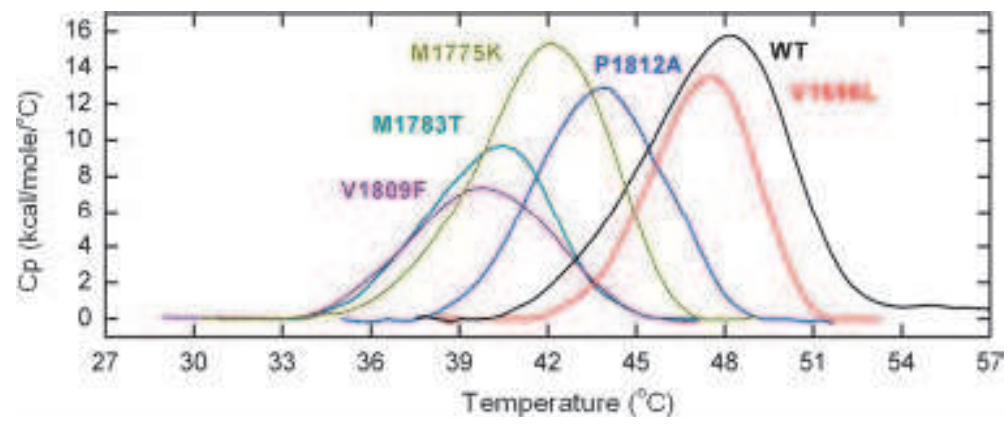

Fig. 3. DSC profiles for the thermally induced denaturation of BRCT-wt and of five missense variants V1809F, M1783T, M1775K, P1812A, and V1696L. (from Drikos et al., 2009).

$\mathrm{V} 1809 \mathrm{~F}$ is a rare mutation linked to hereditary breast/ ovarian cancer. Only a few cases of the mutation have been submitted to the BIC database with loss of function reported by in vitro experiments, regarding the interactions with synthetic phosphopeptides pBACH1/BRIP1 and pCtIP. The residue Val1809 is conserved among species. Val1809 and Met1775 are crucial for the integrity of phosphopeptide binding pocket of the BRCA1 protein and exhibit no binding to either pBACH1/BRIP1 or pCtIP synthetic phosphopeptides. These results, in combination with the fact that V1809F resembles structural destabilization of the native fold similar to M1775R, strongly supports the classification of $\mathrm{V} 1809 \mathrm{~F}$ as pathogenic. 
Additionally, the variant M1652I is located at the first tandem of the BRCT domain and seems to have neutral influence on breast cancer pathogenesis. Based on preliminary structural studies by our laboratory, this variant is less involved in structural alteration of BRCT but further analysis is required. M1652I is classified as low risk mutation. Therefore we decided to include it to our study in order to compare it with more destabilizing mutants such as V1809F and M1775K.

In order to assess how the selected BRCA1-BRCT mutants influence the subcellular localization of BRCA1, we produced BRCA1-GFP fusion proteins with the corresponding mutations introduced at the BRCT domain. The GFP-BRCA1-BRCT mutated proteins were inserted into MCF-7 cells and their subcellular localization was assessed by fluorescent microscopy.

According to our results, destabilizing mutations of the carboxyl terminal region of BRCA1 seem to influence protein localization and presumably DDR. As shown in Fig 4, BRCA1V1809F-GFP and M17775K are restricted to the cytoplasm in contrast to the nuclearcytoplasmic localization of BRCA1wt and M1652I. As EGFP-BRCA1-M1652I shows similar subcellular distribution to the BRCA1wt-GFP protein (detected both in the nucleus and the cytoplasm), it is presumed that the structural change caused by replacement of M1652 to Ile has a minor effect of BRCA1 nuclear transport. UV irradiation of cells expressing wt or the mutants mentioned failed to drive $\mathrm{M} 1175 \mathrm{~K}$ and $\mathrm{V} 1809 \mathrm{~F}$ to the cell nucleus in contrast to both wt and M1652I which were then detected exclusively in the nucleus and shown to at least in part colocalize with Rad51 foci (data not shown from Drikos et al., submitted). Mutations such as M1775K and V1809F which disrupt BRCA1 C-terminal folding, appears that result to conformational and functional changes which might be restrictive to BRCA1 nuclear transport in contrast to more mild missense variants such as M1652I. These results suggest that structural integrity modifications of the BRCA1-BRCT domain can be reflected to the protein's subcellular localization and therefore can serve for further characterization and classification of the variant, in combination with the structural data (table 1). M1175K and V1809F are located near to the binding site of the inter-repeat region and affect through hydrophobic interactions the structural and functional integrity of the domain.

\begin{tabular}{|lcccccc|}
\hline & $\begin{array}{c}\text { Effect of } \\
\text { mutation }\end{array}$ & $\begin{array}{c}\text { Cancer } \\
\text { Risk of the } \\
\text { mutation }\end{array}$ & $\begin{array}{c}\text { Structural } \\
\text { Stability }\end{array}$ & $\begin{array}{c}\text { Functional } \\
\text { Activity with } \\
\text { synthetic } \\
\text { peptides }\end{array}$ & $\begin{array}{c}\text { Subcellular } \\
\text { localization } \\
(-U V)\end{array}$ & $\begin{array}{c}\text { Subcellular } \\
\text { localization } \\
\text { (+UV) }\end{array}$ \\
\hline $\begin{array}{l}\text { GFP- } \\
\text { BRCA1- } \\
\text { V1809F }\end{array}$ & $\begin{array}{c}\text { Potential } \\
\text { hydrophobic }\end{array}$ & Deleterious & Destabilizing & $\begin{array}{c}\text { Alter binding } \\
\text { affinity }\end{array}$ & Cytoplasmic & Cytoplasmic \\
\hline $\begin{array}{l}\text { GFP- } \\
\text { BRCA1- } \\
\text { M1775K }\end{array}$ & $\begin{array}{c}\text { Potential } \\
\text { hydrophobic }\end{array}$ & Deleterious & Destabilizing & $\begin{array}{c}\text { Alter binding } \\
\text { affinity }\end{array}$ & Cytoplasmic & Cytoplasmic \\
\hline $\begin{array}{l}\text { GFP- } \\
\text { BRCA1- } \\
\text { M1652I }\end{array}$ & No effect & Neutral & No effect & Unknown & Nuclear- & Cytoplasmic \\
\hline $\begin{array}{l}\text { GFP- } \\
\text { BRCA1wt }\end{array}$ & & & & Nuclear- \\
\hline
\end{tabular}

Table 1. Summary of the impact of BRCA1-BRCT mutants on the structural, functional and cellular levels. Mutations such as M1775K and V1809F, which disrupt BRCA1 C-terminal folding, induce also alterations of the integrity of the BRCA1-BRCT domain and the proteins subcellular localization. 


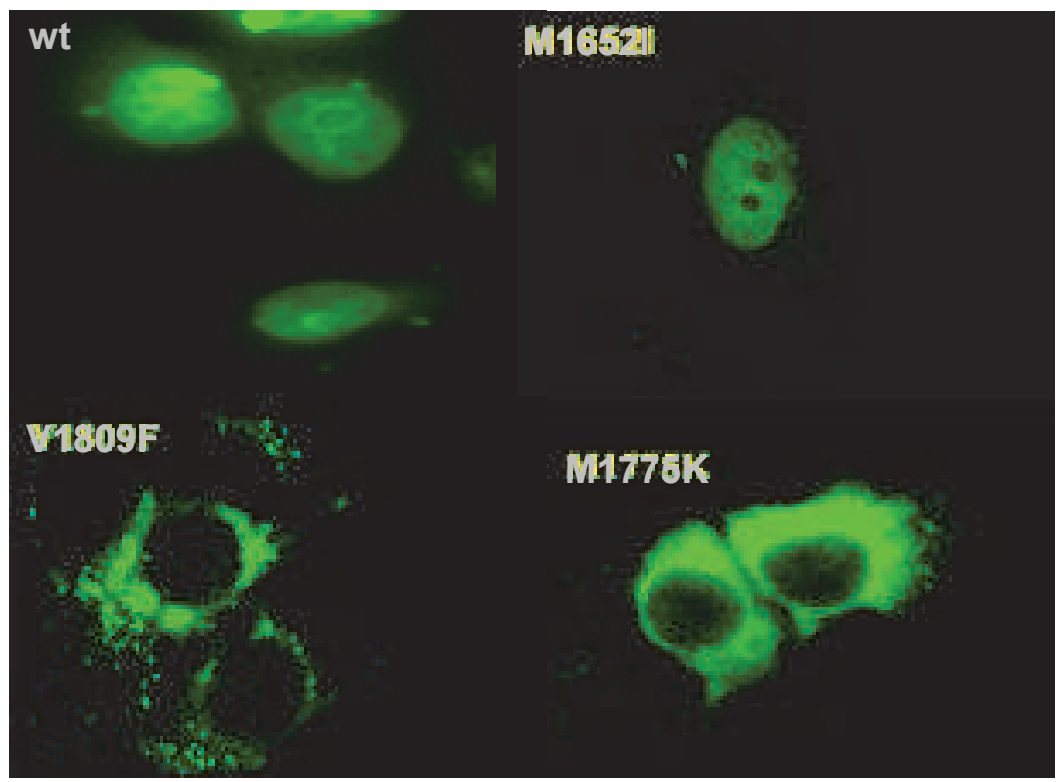

Fig. 4. Subcellular mis-localization of cancer linked GFP-BRCA1-BRCT mutations, M1775K and V1809F in the cytoplasm of MCF-7 cells in contrast to the wild type BRCA1 (wt) and the 'neutral' mutation of BRCT, M1652I which are detected in both the nucleus and the cytoplasm.

A living cell is a dynamic unit with flexible equilibrium between different processes which drive cell fate and determination decisions. The various pathways involved are either activated or suppressed as a result of qualitative and /or quantitative interactions between biomolecules. BRCA1 is an elegant paradigm of both kinds of interactions. Truncated or absent BRCA1 (abolishment of qualitative interactions) leads to impaired DNA repair, carcinogenesis and cancer progression. As indicated by the studies of various missense mutations there are cases where although the mutated BRCA1 seems to function properly, a significant proportion of BRCA1 molecules adopts an unfolding state and only few molecules are found in a given time in proper and functional structure. M1324K, R42573L mutations are good paradigms where biophysical studies of these BRCA1 mutants indicated that although interactions with phosphopeptides were attained, the majority of mutant molecules were detected in improper folding state. Moreover, as many missense variants remain to be characterized, combination of biophysical with cell/molecular biology studies, as in the case of M1775K and V1809F mutations, is expected to substantially contribute in their classification regarding to cancer-relation.

\subsection{The RAD51 structure - function relation paradigm}

DNA double strand breaks (DSBs), produced by either exogenous causes or in order to restore stalled replication forks during genome replication, are detrimental to cell survival and are mainly restored by either Homologous Recombination Repair (HRR) or NonHomologous End Joining (NHEJ). The two pathways compete for each other while a number of factors such as cell cycle stage and availability of duplicated DNA regulate the 
final choice. HRR is the prominent (high fidelity) DSB repair pathway, requiring an homologous DNA sequence present (the identical sister chromatid located in proper distance) and therefore is mainly active during S/G2 phases of cell cycle, while the error prone NHEJ pathway is mainly active during G1 and early S phase, although in certain cases can work in all cell cycle phases. HRR compete NHEJ pathway through a complicated manner, where initial DNA damage signalling factors play important roles. Damage processing and effectiveness of repair are incessantly checked by sensor molecules which through a series of distinct but interconnected pathways prolong cell cycle arrest, induce senescence or apoptosis depending on the information flow and the signals produced (Freeman \& Monteiro 2010).

An important early step in HRR is the conversion of double to single stranded DNA in the area of the double strand break, which in turn is coated by the Replication Protein A (RPA) and can be extended up to $4-5 \mathrm{~Kb}$ on both sides along the break point. Displacement of RPA by RAD51, assembled as a nucleoprotein filament on the ssDNA, is the initial step towards HRR and is highly regulated through interactions with a variety of accessory proteins referred to as the 'recombination mediators' (Essers et al., 2002; Henning \& Sturzbecher 2003; Schild \& Wiese, 2010; Forget \& Kowalczykowski, 2010; West, 2003; San Filippo et al., 2008; Li \& Heyer, 2008). The central event in HRR is the synapsis of the singlestranded (ss)DNA molecule - produced along the double strand break point - with homologous duplex DNA. The strand invasion is mediated by the strand transferase RAD51 oligomerized on ssDNA as an active nucleoprotein filament (and the corresponding cofactors needed for filament assembly and function), which initiates the strand exchange that leads to recombination. RAD51, a recombinase essential for cell viability, is one of the most conserved molecules known. RAD51 mediates strand exchange via distinct reactions grouped into the presynaptic, synaptic, and postsynaptic phases (2) (Heyer, 2007; Shivji et al., 2009). The major steps of HRR process are schematically illustrated in Scheme 3.

RAD51 assembly on ssDNA and subsequent catalysis of homology dependent strand invasion is mainly driven by the tumor suppressor protein BRCA2 while during the different phases of HRR RAD51 interacts sequentially with other molecules involved in HRR, cell cycle control and cell fate decisions. RAD51, as part of dynamic structures called DNA damage foci, seems to be a stably associated core component, whereas other co-factors such as Rad52 and Rad54 rapidly and reversibly interact with the structure (Essers et al., 2002). RAD51 function depends on protein re-localization and is mainly regulated by various post-translational modifications, mainly phosphorylation (Slupianek et al., 2001; Venkitaraman, 2001), as well as non-covalent interaction with SUMO (Ouyang et al., 2009). Along evolution recombinase molecules are highly conserved, starting from the prokaryotic orthologue RecA to mammalian RAD51. The fact the RecA seems not to be an ancestor of RAD51 but these two molecules are considered to have evolved by converging evolution, suggests that the structure obtained is crucial for the specific recombination function and cannot afford modifications. This notion is further supported by the absence of RAD51 coding region mutants in any cancer type, while cells or animals that do not express RAD51 eventually are not viable (Tsuzuki et al., 1996; Sonoda et al., 1998). Despite the non-detection of RAD51 mutants itself it is clear that mutations in recombination mediators and comediators, which control RAD51 activity and availability, are highly related to cancer susceptibility and particularly breast cancer (Venkitaraman, 2009; Rahan et al., 2007; Seviour $\&$ Lin, 2010). Additionally, the 'guardian of the genome' p53, found mutated in more than $50 \%$ of cancers, also directly interacts with RAD51 presumably connecting HRR efficiency to 
cell cycle control and apoptosis (Henning \& Sturzbecher, 2003; Gatz \& Wiesmuller, 2006; Lazao-Trueba \& Silva, 2006).

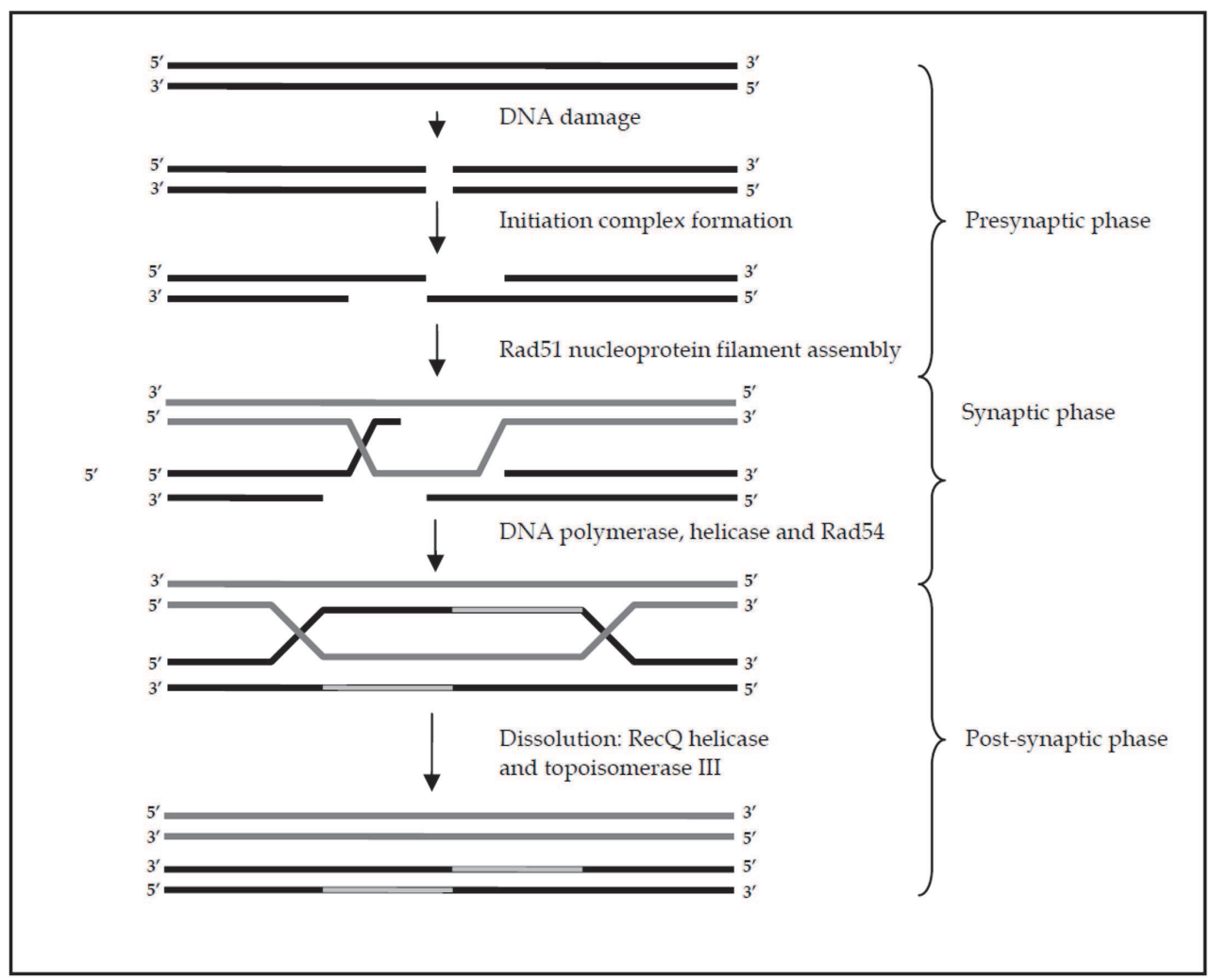

Scheme 3. Simplified description of HRR major steps. The presynaptic phase involves resection of the $5^{\prime}$ terminated strand at the DNA double strand break point (black line) and the formation of the RAD51 active nucleoprotein filament on 3'ssDNA tails. During the synaptic phase RAD51 traces the homologous strand (grey line)(usually the sister chromatid) and performs the strand exchange. After DNA heteroduplex extension and branch migration (newly synthesized DNA is shown as framed grey line) the Holiday junctions produced are separated resulting in two intact homologous DNA molecules. (Resolution or dissolution of holiday junction may also involve crossing over resulting in chimeric but still homologous DNA molecules).

RAD51 function is mainly controlled by the breast cancer susceptibility gene 2 product BRCA2 which acts as a recombination mediator (scheme 4). Briefly, BRCA2 targets RAD51 to ssDNA for assembly into a nucleoprotein filament, stabilizes the ATP-bound form of RAD51 and inhibits RAD51 assembly on dsDNA (Shivji et al., 2009). BRCA2 is an extremely large protein of 3418 residues and essentially contributes to RAD51-mediated HRR through several regions. RAD51 interacts with 8 copies of $\sim 35$ residues repeated motifs (BRC repeats) located at exon 11 (Yu et al., 2003), as well as with an unrelated carboxyl-terminal motif in exon 27 (Esashi et al., 2007). The BRC repeats sequence, unlike the C-terminal motif, is 
evolutionarily conserved. RAD51 replaces RPA in ssDNA, a process regulated by the DNAbinding domain of BRCA2, in cooperation with the BRC repeats and the contribution of other RAD52 epistasis group members as Rad52 and Rad54. BRC repeats of BRCA2 bind to the core of RAD51 by mimicking the structure of an adjacent Rad51 monomer (Pellegrini at al., 2002). RAD51 loading on ssDNA is promoted by the BRCA2[BRC1-8] region while RAD51 assembly on dsDNA is at the same time suppressed. This way the efficiency of RAD51-mediated HRR is further enhanced. RAD51 function can either be stimulated or suppressed by activities of the BRC repeats, depending on the experimental conditions used and the BRC: RAD51 molar ratio used (Galkin et al., 2005; Shivji et al., 2009; Carreira et al., 2009; Rajendra \& Venkitaraman, 2010). BRC4 also blocks nucleation of RAD51 onto dsDNA while not disassembling Rad51-dsDNA filaments. (Carreira A, et al., 2009). At lower molar ratios BRC3 or BRC4 actually bind and form stable complexes with RAD51-DNA nucleoprotein filaments. Only at high concentrations of the BRC repeats are filaments disrupted. The specific protein-protein contacts occur in the RAD51 filament by means of the N-terminal domain of RAD51 for BRC3 and the nucleotide-binding core of RAD51 for BRC4 (Galkin et al., 2005; Rajendra \& Venkitaraman 2009). These observations show that the BRC repeats bind distinct regions of RAD51 and are nonequivalent in their mode of interaction. These results might explain how disruption of a single RAD51 interaction site in BRCA2 might modulate the ability of RAD51 to promote recombinational repair and lead to an increased risk of breast cancer. Moreover, the dysregulated molar ratio present in a cell may drive hyper-recombination effects leading to abnormal outcome and in part may explain why mutations in BRCA2 predispose individuals to breast cancer, a consequence of the role of BRCA2 in DNA repair.

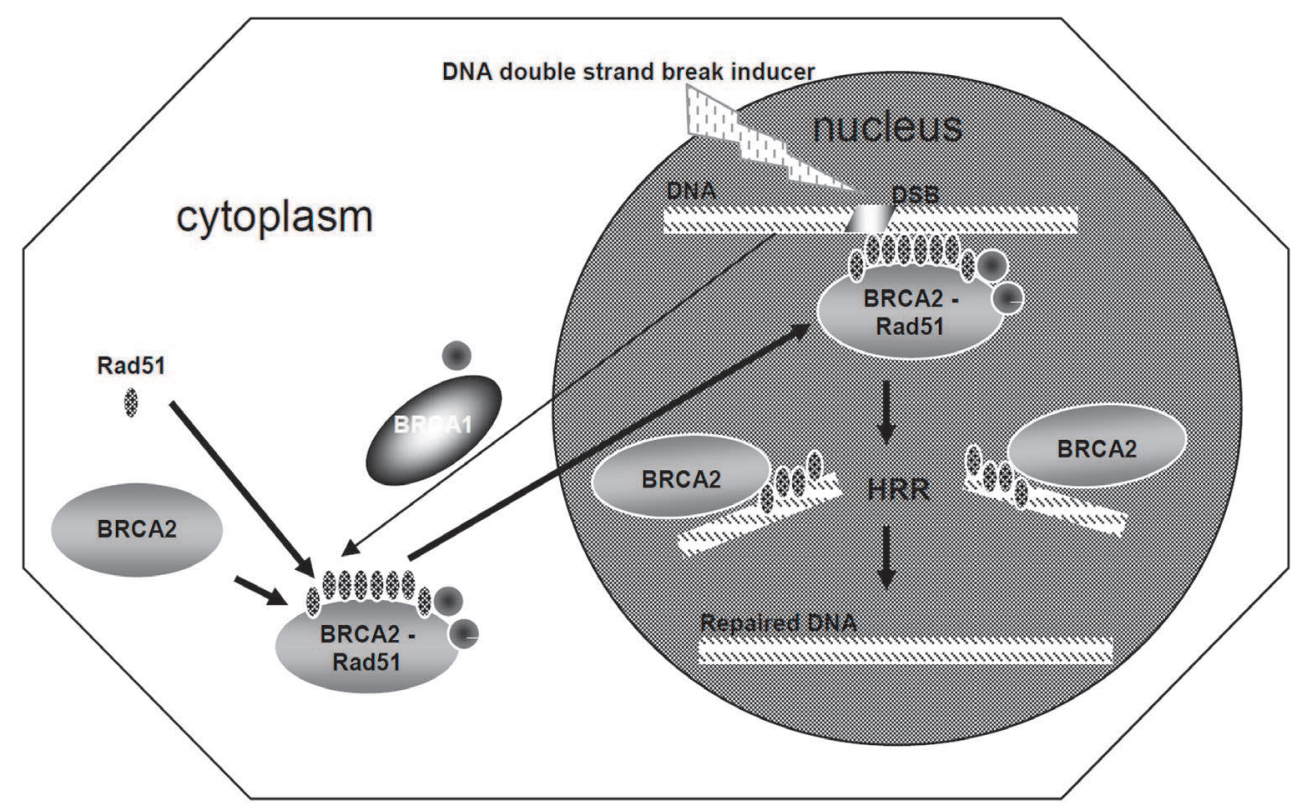

Scheme 4. Simplistic cartoon of the HRR process depicting Rad51 functions. 
RAD51 filaments are further stabilized by direct interaction of the BRCA2 C terminus to the interface created by two adjacent RAD51 protomers. This way filaments cannot be dissociated by the BRC repeats. Interaction of the BRCA2 $\mathrm{C}$ terminus with the RAD51 filament causes a large movement of the flexible RAD51 N-terminal domain that is important in regulating filament dynamics. RAD51 interaction with the BRCA2 C-terminal region may facilitate efficient nucleation of RAD51 multimers on DNA and thereby stimulate recombination-mediated repair. (Esashi et al., 2007). Data from studies the Caenorhabditis elegans BRCA2 homolog CeBRC-2 support a model where an interaction with RAD-51 alone is likely involved in filament nucleation, whereas a second independent interaction is involved in in situ stabilization of RAD51 filaments by BRCA2 and provide further insight into why mutations in many different positions within BRCA2 lead to loss of genomic stability (Petalcorin et al., 2007).

RAD51, the central homology strand search and strand exchange effector in HRR can serve as a nice example to show how unregulated protein levels can abolish normal cell fate decisions and result in premature ageing or malignancies, depending on the mechanisms involved. RAD51 is one of the most conserved proteins known and essential for cell survival (Henning \& Sturzbecher, 2003; Sonoda et al., 1998). While no mutations have ever been detected in human cancers, in many tumors significantly up- or downregulated levels of RAD51 have been observed (Maacke et al., 2000; Henning \& Sturzbecher 2003; Klein, 2008). Moreover, high-level expression of RAD51 is an independent prognostic marker of survival in non-small-cell lung cancer patients (Qiao et al., 2005). In addition, haematopoietic progenitor cells, when Rad51 is overexpressed showed elevated levels of chromosomal alterations, similar to those observed in tumors of the hematopoietic system (Francis and Richardson, 2007). Notably, both positive and negative regulation of HRR is required in order to maintain genomic stability with precise repair and suppression of deleterious rearrangements. The only tumorigenesis-related variation found in the rad51 gene is a G->C change in the $5^{\prime}$ untranslated rad51 mRNA region. This variation has been correlated to higher risk for breast cancer in BRCA2 mutation carriers and is possibly involved in mRNA modified translation capability resulting in abnormal RAD51 protein levels (Antoniou et al., 2007).

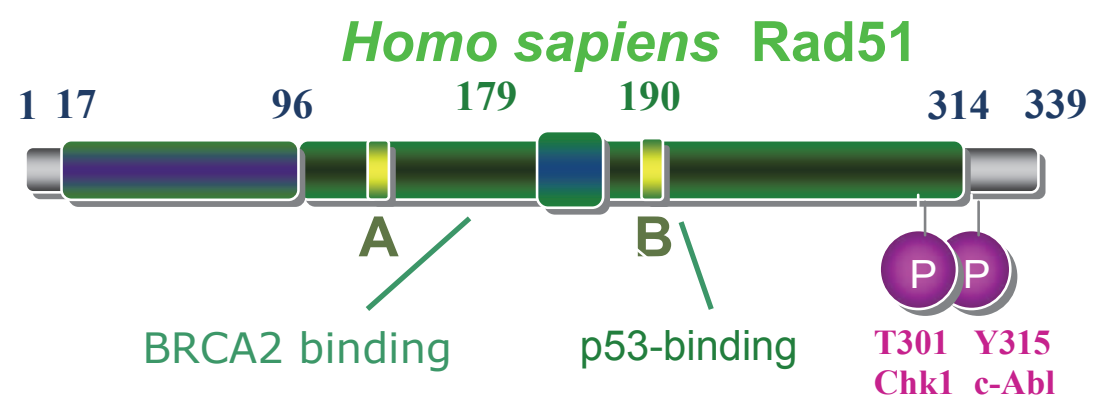

Scheme 5. Scematic representation of human Rad51 protein. The areas responsible for interaction with p53 and BRCA2 are indicated. 
Since Rad51 overexpression can compensate for loss of function of other key molecules of DDR, including BRCA1 and BRCA2, experimental evidences from various research groups support two models: 1 . Rad51 abnormal levels lead to genomic instability early in cancer development, thereby placing Rad51 modified expression as a leading cause of transformation and 2. Rad51 overexpression can protect cancer cells from DNA damage as more effective repair occurs further stabilizing the neoplastic clone and render it more aggressive and metastatic (Schild \& Wiese, 2010). As cancer is an extremely complex set of diseases and can develop by different aetiologies while achieving similar phenotype of independent and uncontrolled growth the two models presented by Schild and Wiese can each adequately explain the neoplastic procedure of different cancer types.

The exact causes of Rad51 overexpression are still poorly explored but there is a number of data indicating both transcriptional regulation and protein stability and turnover modification. p53, the tumor suppressor that is implicated in DNA repair control, is involved in transcriptional regulation of rad51 (Arias-Lopez et al., 2006). p53 is mutated in about half of human cancers resulting in loss of suppression of rad51 transcriptional regulation. Notably, as p53 directly interacts with Rad51 (Stürzbecher et al., 1996), in cases of p53 mutations inhibition of Rad51 activity could be abolished. Moreover, in cases of either TP53 deletion or some TP53 point mutations Rad51 expression up-regulation is detected. The Transcriptional activator protein 2 (AP2), in combination with p53 suppresses rad51 transcription (Hannay et al., 2007). Abl kinase phosphorylates Rad51 in Tyr315 and in cases of the presence of the oncogenic constituvely active BCR/Abl fusion tyrosine kinase (i.e. in Ph+ leukaemias) Rad 51 expression is increased (Slupianek et al., 2001).

Aiming in further clarifying aspects of structure-function relationship of RAD51, we produced several RAD51 mutants by altering amino acid residue candidates to be involved in RAD51-BRCA2 or RAD51-p53 interaction (fig 5). Exogenous expression the RAD51m6 mutant, fused to EYFP, altered their subcellular localization compared to the wt protein.

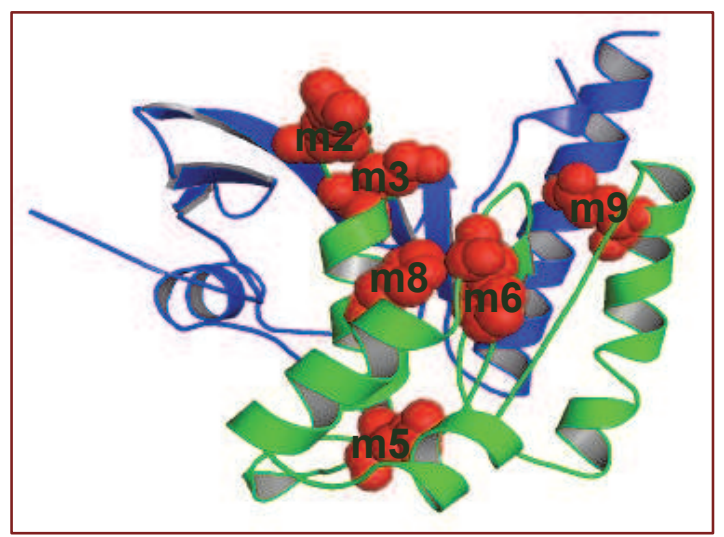

Fig. 5. Solved structure of Rad51-BRC4 complex (adapted from PDB: 1NOW, (Pellegrini et al., 2002)) where candidate residues presumed to alter Rad51-BRCA2/p53 complex interaction are indicated. In vitro Site-Directed mutagenesis was employed in order to alter each indicated residue to Ala. Mutant form positions are indicated. 


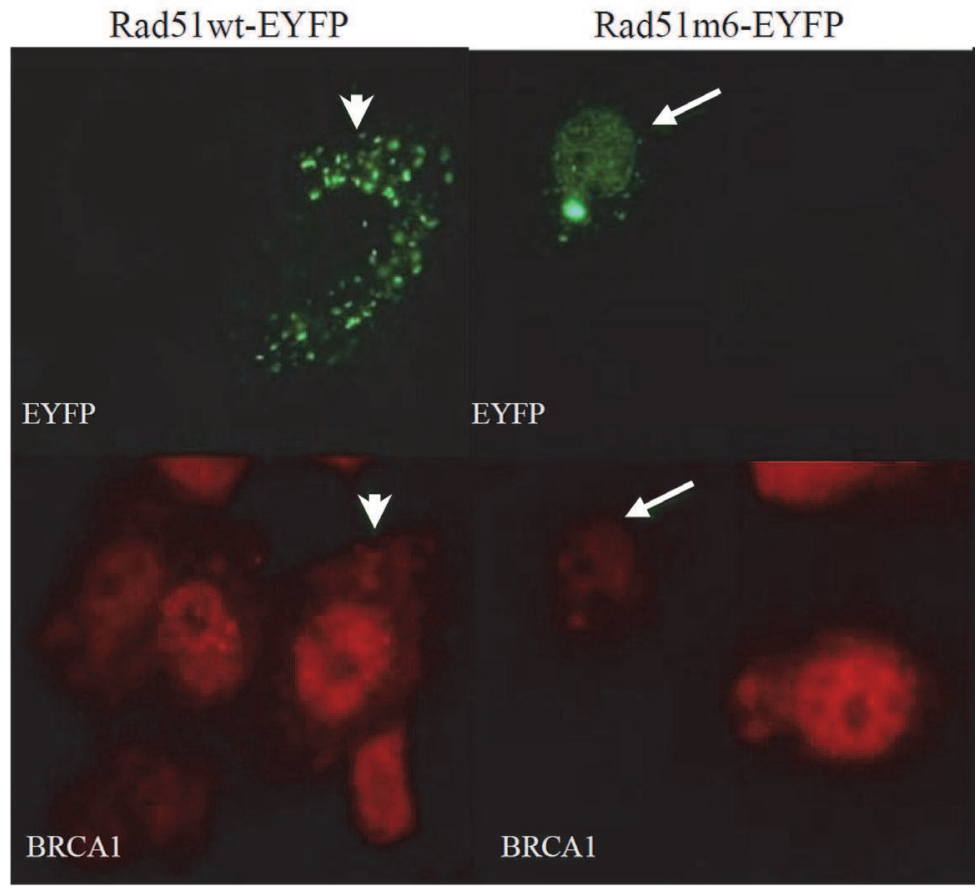

Fig. 6. MCF7 cells expressing exogenous Rad51wt-EYFP and Rad51m6-EYFP as indicated. Endogenous BRCA1 expression, as detected by immunofluoresence, shows a significant reduction in all cells expressing the Rad51m6-EYFP in comparison to both the Rad51wt-EYFP expressing as well as to non-transfected cells (Boutou et al., unpublished data).

Moreover cells expressing RAD51m6 showed a modification in their cell cycle progression (data not shown) accompanied by modifications in expression of BRCA1 (fig 6), p53 and p21waf1 (data not shown). Notably, RAD51m6 electively kill certain cancer cell lines as HeLa cells, but do not affect the Caspase 3 defective MCF-7 cells.

Double strand breaks (DSBs) of DNA is the most deleterious damage of the genome since if not repaired accurately can result in ICL, translocations, chromatin rearrangements, $\mathrm{LOH}$ and mutation accumulation. HRR restores DNA damage in mitotic cells by gene conversion, where the broken sequence is converted to the sequence of the repair template (original sequence), which remains unaltered. In case of HRR misregulation other templates can be used including homologous chromosomes and repetitive elements on heterologous chromosomes. Such data indicate that single amino acid residue alterations of Rad51 are capable to modify the behaviour of the entire protein, presumably through structural modifications. These results, combined with the fact that RAD51 protein in nature is not mutated, suggest that its proper function is strongly dependent on its high degree of structural conservation throughout evolution. Moreover, the combination of the active peptide of such mutants under the promoter of human RAD51 which in the absence of the N-terminal region of Rad51 enhances expression up to 10 fold in cancer cell lines, could serve as a potential anti-cancer agent, selectively targeting malignant cells. 


\section{Conclusions}

In conclusion maintenance of genome integrity depends on structure-function relationship of the protein molecules involved. Proper response to DNA damage mainly relies on functional components of DDR driving their appropriate complex formation with partner proteins. These processes are regulated by a number of post-translational modifications, distinct protein isoforms and protein availability (stability / degradation). In case these interactions are deregulated due to genetic / epigenetic causes a balanced cell cycle progression and cell fate determination are abolished in favour of cancer/ageing. Structural / biophysical data accompanied by functional experiments of key DNA repair molecules are significant for: (a) elucidating which residues or structural elements are really necessary for proper function at the molecular level, (b) asses/classify variants identified in individuals, (c) enriching diagnostic markers in cancer and (d) designing effective small molecules to target protein molecules essential for cell survival and genome integrity.

Co-operation of various disciplines is a fundamental prerequisite for fulfilling such a vision, and numerous attempts worldwide work on this subject with promising results.

\section{References}

Arias-Lopez C., Lazaro-Trueba I., Kerr P., Lord C.J., Dexter T., Iravani M., Ashworth A. and Silva A. p53 modulates homologous recombination by transcriptional regulation of the RAD51 gene. EMBO Rep. 2006 7: 219-224

Best B.P. Nuclear DNA damage as a direct cause of aging. Rejuvenation Research 2009, 12: 199-208.

Buchhop S., Gibson M.K., Wang X.W., Wagner P., Sturzbecher H-W. and Harris C.C. Interaction of p53 with the human Rad51 protein. Nucleis Acids Res. 1997 25: 3868-3874.

Carreira A, Hilario J, Amitani I, Baskin RJ, Shivji MK, Venkitaraman AR, Kowalczykowski SC. The BRC repeats of BRCA2 modulate the DNA binding selectivity of RAD51. Cell 2009 136:1032-1043.

Deng C.-X. BRCA1: cell cycle checkpoint, genetic instability, DNA damage response and cancer evolution. Nucleic Acids Res., 2006, 34: 1416-1426

Drikos I. Nounesis G. and Vorgias C.E. Characterization of cancer-linked BRCA1-BRCT missense variants and their interaction with phosphoprotein targets. Proteins. 2009 77: 464-76.

Esashi F, Galkin VE, Yu X, Egelman EH, West SC. Stabilization of RAD51 nucleoprotein filaments by the C-terminal region of BRCA2. Nat Struct Mol Biol. 2007 14:468-74.

Essers J., Houtsmuller A., van Vielen L., Paulusma C., Nigg A., Pastink A., Vermeulen W., Hoeijmakers J. and Kanaar R. Nuclear dynamics of RAD52 group homologous recombination proteins in response to DNA damage. EMBO J 200221 2030-37

Feldser D.M., Greider C.W. Short telomeres limit tumor progression in vivo by inducing senescence. Cancer Cell. 2007 11:461-9.

Forget A. \& Kowalczykowski S.C. Single-molecule imaging brings Rad51 nucleoprotein filaments into focus. TICS 2010 20: 269-76

Francis R, Richardson C. Multipotent hematopoietic cells susceptible to alternative doublestrand break repair pathways that promote genome rearrangements. Genes Dev. 2007 21:1064-74.

Freeman A. and Monteiro A. Phosphatases in the cellular response to DNA damage. Cell Comm.Signaling 2010, 8:27-38

Friedberg EC, Walker GC, Siede W, Wood RD, Schultz RA, Ellenberger T. (2006). DNA Repair and Mutagenesis, part 3. ASM Press. 2nd edition 
Galkin V. E., Esashi F., Yu X. , Yang S., West S. C., and Egelman E. H. BRCA2 BRC motifs bind RAD51-DNA filaments. PNAS 2005, 102: 8537-8542

Gatz S.A \& Wiesmuller L p53 in recombination and repair. Cell Death Differ. 2006 13: 1003-1016

Glover JN, Insights into the molecular basis of human hereditary breast cancer from studies of theBRCA1 BRCT domain. Familial Cancer 2006 5: 89-93

Gough CA, Gojobori T, Imanishi T. Cancer-related mutations in BRCA1-BRCT cause longrange structural changes in protein-protein binding sites: a molecular dynamics study. Proteins, 2007 66:69-86.

Hannay J.A., Liu J., Zhu Q.S., Bolshakov S.V., Li L., Pisters P.W., Lazar A.J., Yu D., Pollock R.E. and Lev D. Rad51 overexpression contributes to chemoresistance in human soft tissue sarcoma cells: a role for p53/activator protein 2 transcriptional regulation. Mol. Cancer Ther. 2007 6: 1650-1660.

Henning W, Sturzbecher HW. 'Homologous recombination and cell cycle checkpoints: Rad51 in tumour progression and therapy resistance'. Toxicology. 2003, 193: 91-109.

Heyer W.-D. Biochemistry of eukaryotic homologous recombination. Top Curr Genet. 2007, 17: $95-133$

Klein H. The consequences of Rad51 overexpression for normal and tumor cells. DNA Repair (Amst) 2008 7: 686-693.

Lazao-Trueba I. Arias. C. \& Silva A. Double bolt regulation of Rad51 by p53: a role for transcriptional repression. Cell Cycle 2006 5: 1062-1065

Lee MS, Green R, Marsillac SM, Coquelle N, Williams RS, Yeung T, Foo D, Hau DD, Hui B, Monteiro AN, Glover JN. Comprehensive analysis of missense variations in the BRCT domain of BRCA1 by structural and functional assays. Cancer Res. 2010 70:4880-90.

Li X \& Heyer WD. Cell Res 2008 Homologous recombination in DNA repair and DNA damage tolerance. 18: 99-113

Lindahl T. Instability and decay of the primary structure of DNA. Nature. 1993, 362: 709-715

Lisby M \& Rothstein R. Localization of checkpoint and repair proteins in eukaryotes. Biochimie 2005, 87: 579-89.

Lodish H, Berk A, Matsudaira P, Kaiser CA, Krieger M, Scott MP, Zipursky SL, Darnell J. (2004). Molecular Biology of the Cell, p963. WH Freeman: New York, NY. 5th ed.

Maacke H, Opitz S, Jost K, Hamdorf W, Henning W, Kruger S, Feller AC, Lopens A, Diedrich K, Schwinger E, Sturzbecher HW. 'Over-expression of wild-type Rad51 correlates with histological grading of invasive ductal breast cancer'. Int J Cancer. 2000, 88: 907-13.

Mark W.Y. Liao J.C, Lu Y., Ayed A, Laister R, Szymczyna B, Chakrabartty A, Arrowsmith $\mathrm{CH}$. Characterization of segments from the central region of BRCA1: an intrinsically disordered scaffold for multiple protein-protein and protein-DNA interactions? J. Mol.Biol. 2005, 345: 275-87

Morita R, Nakane S, Shimada A, Inoue M, Iino H, Wakamatsu T, Fukui K, Nakagawa N, Masui R, Kuramitsu S. Molecular mechanisms of the whole DNA repair system: a comparison of bacterial and eukaryotic systems. J Nucleic Acids. 2010:179594.

Murphy CG, Moynahan ME. BRCA gene structure and function in tumor suppression: a repair-centric perspective. Cancer J. 2010 16: 39-47.

Ouchi T. BRCA1 Phosphorylation. Biological Consequences. Cancer Biol. Ther. 2006, 5: 470-475

Ouyang KJ, Woo LL, Zhu J, Huo D, Matunis MJ, Ellis NA. 2009 SUMO modification regulates BLM and RAD51 interaction at damaged replication forks. PLoS Biol. 7 e1000252

Paull TT, Cortez D, Bowers B, Elledge SJ, Gellert M. 2001, Direct DNA binding by Brca1. Proc Natl Acad Sci U S A. 98 6086-91

Pellegrini L, Yu DS, Lo T, Anand S, Lee M, Blundell TL, Venkitaraman AR. Insights into DNA recombination from the structure of a RAD51-BRCA2 complex. Nature. 2002 420: 287-93. 
Petalcorin MI, Galkin VE, Yu X, Egelman EH, Boulton SJ. 2007 Stabilization of RAD-51-DNA filaments via an interaction domain in Caenorhabditis elegans BRCA2. Proc Natl Acad Sci U S A. 104: 8299-8304

Qiao GB, Wu YL, Yang XN, Zhong WZ, Xie D, Guan XY, Fischer D, Kolberg HC, Kruger S, Stuerzbecher HW. High-level expression of Rad51 is an independent prognostic marker of survival in non-small-cell lung cancer patients. Br J Cancer. 2005, 93:137-43.

Rahan R et al., Links between DNA double strand break repair and breast cancer: accumulating evidence from both familiar and nonfamilial cases. Cancer Lett. 2007 248: 1-17

Rajendra E. and Venkitaraman A. R. Two modules in the BRC repeats of BRCA2 mediate structural and functional interactions with the RAD51 recombinase. Nucleic Acids Res. 2010, 38: 82-96

Rogakou E. Megabase chromatin domains involved in DNA double strand breaks in vivo. J. Cell Biol. 1999, 146 905-916

Rowling PJ, Cook R, Itzhaki LS. 2010 Toward classification of BRCA1 missense variants using a biophysical approach. J Biol Chem. JBC 285(26): 20080-20087

San Filippo J, Sung P, Klein H. Mechanism of eukaryotic homologous recombination. Annu Rev Biochem. 200877229

Schild D, Wiese C. Overexpression of RAD51 suppresses recombination defects: a possible mechanism to reverse genomic instability. Nucleic Acids Res. 201038 1061-70

Seviour E \& Lin S-Y 2010 Aging 2 900-907 The DNA damage response: Balancing the scale between cancer and ageing;

Shivji MK, Mukund SR, Rajendra E, Chen S, Short JM, Savill J, Klenerman D, Venkitaraman AR. The BRC repeats of human BRCA2 differentially regulate RAD51 binding on single- versus double-stranded DNA to stimulate strand exchange. Proc Natl Acad Sci U S A. 2009106 13254-13259

Slupianek A. Schmutte C., Tombline G., Nieborowska-Skorska M., Hoser G., Nowicki M.O. Pierce A.J. Fishel R. and Skorski T. BCR/ABL regulates mammalian RecA homologs, resulting in drug resistance. Mol. Cell 2001 8: 795-806.

Sonoda E, Sasaki MS, Buerstedde JM, Bezzubova O, Shinohara A, Ogawa H, Takata M, Yamaguchi-Iwai Y, Takeda S. Rad51-deficient vertebrate cells accumulate chromosomal breaks prior to cell death. 1998 EMBO J 17 598-608

Starita LM, Parvin JD. The multiple nuclear functions of BRCA1: transcription, ubiquitination and DNA repair. Curr Opin Cell Biol 2003, 15: 345-50

Stürzbecher HW, Donzelmann B, Henning W, Knippschild U, Buchhop S. p53 is linked directly to homologous recombination processes via RAD51/RecA protein interaction. EMBO J. 1996 15:1992-2002.

Tsuzuki T, Fujii Y, Sakumi K, Tominaga Y, Nakao K, Sekiguchi M, Matsushiro A, Yoshimura Y, MoritaT. Targeted disruption of the Rad51 gene leads to lethality in embryonic mice. Proc Natl Acad Sci U S A. 1996 93: 6236-6240

Venkitaraman A.R. Linking the cellular functions of BRCA genes to cancer pathogenesis and treatment. Ann.Rev. Pathol. 2009 4: 461-487

Vigneron A, Vousden KH. p53, ROS and senescence in the control of aging. Aging (Albany NY), 2010 2:471-4.

West SC. Molecular views of recombination proteins and their control. Nat Rev Mol Cell Biol. 2003 4:435-45.

Williams RS, Chasman DI, Hau DD, Hui B, Lau AY, Glover JN. Detection of protein folding defects caused by BRCA1-BRCT truncation and missense mutations. J. Biol. Chem. 2003, 278: 53007-16 


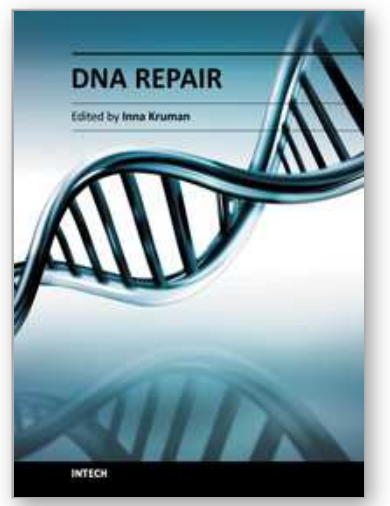

\author{
DNA Repair \\ Edited by Dr. Inna Kruman
}

ISBN 978-953-307-697-3

Hard cover, 636 pages

Publisher InTech

Published online 07, November, 2011

Published in print edition November, 2011

The book consists of 31 chapters, divided into six parts. Each chapter is written by one or several experts in the corresponding area. The scope of the book varies from the DNA damage response and DNA repair mechanisms to evolutionary aspects of DNA repair, providing a snapshot of current understanding of the DNA repair processes. A collection of articles presented by active and laboratory-based investigators provides a clear understanding of the recent advances in the field of DNA repair.

\title{
How to reference
}

In order to correctly reference this scholarly work, feel free to copy and paste the following:

Effrossyni Boutou, Vassiliki Pappa, Horst-Werner Stuerzbecher and Constantinos E. Vorgias (2011). StructureFunction Relationship of DNA Repair Proteins: Lessons from BRCA1 and RAD51 Studies, DNA Repair, Dr. Inna Kruman (Ed.), ISBN: 978-953-307-697-3, InTech, Available from: http://www.intechopen.com/books/dnarepair/structure-function-relationship-of-dna-repair-proteins-lessons-from-brca1-and-rad51-studies

\section{INTECH}

open science | open minds

\section{InTech Europe}

University Campus STeP Ri

Slavka Krautzeka 83/A

51000 Rijeka, Croatia

Phone: +385 (51) 770447

Fax: +385 (51) 686166

www.intechopen.com

\section{InTech China}

Unit 405, Office Block, Hotel Equatorial Shanghai

No.65, Yan An Road (West), Shanghai, 200040, China

中国上海市延安西路65号上海国际贵都大饭店办公楼 405 单元

Phone: +86-21-62489820

Fax: $+86-21-62489821$ 
(C) 2011 The Author(s). Licensee IntechOpen. This is an open access article distributed under the terms of the Creative Commons Attribution 3.0 License, which permits unrestricted use, distribution, and reproduction in any medium, provided the original work is properly cited. 